\title{
Virtual Respiratory Urgent Clinics for COVID-19 Symptoms
}

\author{
Rebecca S. Brienza, MD, MPH; Juliette F. Spelman, MD; Kelly Hager, MD; and Christopher Ruser, MD
}

Background: The COVID-19 pandemic has forced a shift from in-person to virtual care to reduce exposure risks to patients and health care workers. This report aims to describe a large primary care system's implementation of virtual respiratory urgent care clinics (VRUCs).

Methods: The VA Connecticut Healthcare System (VACHS) delivers care to more than 58,000 veterans in at 8 primary care sites. VRUCs were established as part of the VACHS primary care rapid transition to virtual care model. Retrospective analysis and qualitative chart reviews were performed from February 2020 through May 2020 to describe characteristics of patients who received care through the VRUCs.
Results: VRUCs were used by $>445$ patients, $51 \%$ received COVID-19 testing, 10\% tested positive, $5 \%$ were admitted to the hospital, and $18 \%$ had $\geq 1$ subsequent emergency department visits. Chart documentation rates of discussion of isolation precautions, high occupational risk, and goals of care were $71 \%, 25 \%$, and $14 \%$, respectively.

Conclusions: Average wait time for health care provider evaluation was 104 minutes, suggesting VRUCs are an expedient means to provide assessment of COVID-19 symptoms. Use of templated notes may ensure routine counseling about isolation, occupation, and goals of care.
Author affiliations can be found at the end of the article.

Correspondence: Rebecca Brienza (rebecca.brienza@va.gov)

Fed Pract. 2021;38(9). Published online September 12. doi: $10.12788 /$ fp. 0178
V irtual care (VC) has emerged as an effective mode of health care delivery especially in settings where significant barriers to traditional in-person visits exist; a large systematic review supports feasibility of telemedicine in primary care and suggests that telemedicine is at least as effective as traditional care. ${ }^{1}$ Nevertheless, broad adoption of VC into practice has lagged, impeded by government and private insurance reimbursement requirements as well as the persistent belief that care can best be delivered in person. ${ }^{2-4}$ Before the COVID-19 pandemic, states that enacted parity legislation that required private insurance companies to provide reimbursement coverage for telehealth services saw a significant increase in the number of outpatient telehealth visits (about $\geq 30 \%$ odds compared with nonparity states). ${ }^{3}$

With the onset of the COVID-19 pandemic, in-person medical appointments were converted to VC visits to reduce increased exposure risks to patients and health care workers. ${ }^{5}$ Prior government and private sector policies were suspended, and payment restrictions lifted, enabling adoption of VC modalities to rapidly accommodate the emergent need and Centers for Disease Control and Prevention (CDC) recommendations for virtual care. ${ }^{6-11}$

The CDC guidelines on managing operations during the COVID-19 pandemic highlighted the need to provide care in the safest way for patients and health care personnel and emphasized the importance of optimizing telehealth services. The federal government facilitated telehealth during the COVID-19 pandemic via temporary measures under the COVID-19 public health emergency declaration. This included Health Insurance Portability and Accountability Act flexibility to use everyday technology for VC visits, regulatory changes to deliver services to Medicare and Medicaid patients, permission of telehealth services across state lines, and prescribing of controlled substances via telehealth without an in-person medical evaluation. ${ }^{7}$

In response, health care providers (HCPs) and health care organizations created or expanded on existing telehealth infrastructure, developing virtual urgent care centers and telephone-based programs to evaluate patients remotely via screening questions that triaged them to a correct level of response, with possible subsequent virtual physician evaluation if indicated. ${ }^{12,13}$

The Veterans Health Administration (VHA) also shifted to a VC model in response to COVID-19 guided by a unique perspective from a well-developed prior VC experience. ${ }^{14-16}$ As a federally funded system, the VHA depends on workload documentation for budgeting. Since 2015, the VHA has provided workload credit and incentivized HCPs (via pay for performance) for the use of $\mathrm{VC}$, including telephone visits, video visits, and secure messaging. These incentives resulted in higher rates of telehealth utilization before the COVID19 pandemic compared with the private 
sector (with $4.2 \%$ and $0.7 \%$ of visits within the VHA being telephone and video visits, respectively, compared with telehealth utilization rates of $1.0 \%$ for Medicare recipients and $1.1 \%$ in an all-payer database). ${ }^{16}$

Historically, VHA care has successfully transitioned from in-person care models to exclusively virtual modalities to prevent suspension of medical services during natural disasters. Studies performed during these periods, specifically during the 2017 hurricane season (during which multiple VHA hospitals were closed or had limited in-person service available), supported telehealth as an efficient health care delivery method, and even recommended expanding telehealth services within non-VHA environments to accommodate needs of the general public during crises and postdisaster health care delivery. ${ }^{17}$

Armed with both a well-established telehealth infrastructure and prior knowledge gained from successful systemwide implementation of virtual care during times of disaster, US Department of Veterans Affairs (VA) Connecticut Healthcare System (VACHS) primary care quickly transitioned to a VC model in response to COVID-19. ${ }^{16}$ Early in the pandemic, a rapid transition to virtual care (RTVC) model was developed, including implementation of virtual respiratory urgent clinics (VRUCs), defined as virtual respiratory symptom triage clinics, staffed by primary care providers (PCPs) aimed at minimizing patient and health care worker exposure risk.

\section{METHODS}

VACHS consists of 8 primary care sites, including a major tertiary care center, a smaller medical center with full ambulatory services, and 6 community-based outpatient clinics with only primary care and mental health. There are 80 individual PCPs delivering care to 58,058 veterans. VRUCs were established during the COVID-19 pandemic to cover patients across the entire health care system, using a rotational schedule of VA PCPs.

\section{COVID-19 Urgent Clinics Program}

Within the first few weeks of the pandemic, VACHS primary care established VRUCS to provide expeditious virtual assessment of respiratory or flu-like symptoms. Using the es-
TABLE Patient Demographics $(\mathrm{N}=445)$

\begin{tabular}{lc} 
Characteristics & Patients, No. (\%) \\
\hline Male & $367(82.5)$ \\
\hline Female & $78(17.5)$ \\
\hline Age groups, y & \\
$20-29$ & $34(7.6)$ \\
$30-39$ & $74(16.6)$ \\
$40-49$ & $6113.7)$ \\
$50-59$ & $92(20.7)$ \\
$60-69$ & $83(18.7)$ \\
$70-79$ & $85(19.1)$ \\
$80-89$ & $14(3.1)$ \\
$90-99$ & $2(0.4)$ \\
\hline Service periods & \\
Gulf War & $214(48.0)$ \\
Vietnam War & $12027.0)$ \\
Post-Vietnam War & $8719.6)$ \\
Post-Korean War & $10(2.2)$ \\
Korean War & $6(1.3)$ \\
Other & $6(1.3)$ \\
World War II & $2(0.4)$ \\
&
\end{tabular}

tablished telehealth system, the intervention aimed to provide emergent screening, testing, and care to those with potential COVID-19 infections. The model also was designed to minimize exposures to the health care workforce and patients.

Retrospective analysis was performed using information obtained from the electronic health record (EHR) database to describe the characteristics of patients who received care through the VRUCs, such as demographics, era of military service, COVID-19 testing rates and results, as well as subsequent emergency department (ED) visits and hospital admissions. A secondary aim included collection of additional qualitative data via a random sample chart review.

Virtual clinics were established January 22, 2020, and data were analyzed over the next 3 months. Data were retrieved and analyzed from the EHR, and codes were used to categorize the VRUCs.

\section{RESULTS}

A total of 445 unique patients used these clinics during this period. Unique patients were defined as individual patients (some may have used a clinic more than once but were counted only once). Of this group, $82 \%$ were male, 
and $48 \%$ served in the Gulf War era (1990 to present). A total of $51 \%$ of patients received a COVID-19 test (clinics began before wide testing availability), and $10 \%$ tested positive. Of all patients using the clinics, approximately $5 \%$ were admitted to the hospital, and $18 \%$ had at least 1 subsequent ED visit (Table).

A secondary aim included review of a random sample of 99 patient charts to gain additional information regarding whether the patient was given appropriate isolation precautions, was in a high-exposure occupation (eg, could expose a large number of people), and whether there was appropriate documentation of goals of care, health care proxy or referral to social work to discuss advance directives. In addition, we calculated the average length of time between patients' initial contact with the health care system call center and the return call by the PCP (wait time).

Of charts reviewed, the majority $(71 \%)$ had documentation of appropriate isolation precautions. Although 25\% of patients had documentation of a high-risk profession with potential to expose many people, more than half of the patients had no documentation of occupation. Most patients (86\%) had no updated documentation regarding goals of care, health care proxy, or advance directives in their urgent care $\mathrm{VC}$ visit. The average time between the patient initiating contact with the health care system call center and a return call to the patient from a PCP was 104 minutes (excluding calls received after 3:30 PM).

\section{DISCUSSION}

This analysis adds to the growing literature on use of VC during the COVID-19 pandemic. Specifically, we describe the population of patients who used VRUCs within a large health care system in a RTVC. This analysis was limited by lack of available testing during the initial phase of the pandemic, which contributed to the lower than expected rates of testing and test positivity in patients managed via VRUCs. In addition, chart review data are limited as the data includes only what was documented during the visit and not the entire discussion during the encounter.

Several important outcomes from this analysis can be applied to interventions in the future, which may have large public health implications: Several hundred patients who reported respiratory symptoms were expeditiously evaluated by a PCP using VC. The average wait time to full clinical assessment was about 1.5 hours. This short duration between contact and evaluation permitted early education about isolation precautions, which may have minimized spread. In addition, this innovation kept patients out of the medical center, eliminating chains of transmission to other vulnerable patients and health care workers.

Our retrospective chart review also revealed that more than half the patients were not queried about their occupation, but of those that were asked, a significant number were in high-risk professions potentially exposing large numbers of people. This would be an important aspect to add to future templated notes to minimize work-related exposures. Also, we identified that few HCPs discussed goals of care with patients. Given the nature of COVID-19 and potential for rapid decompensation especially in vulnerable patients, this also would be important to include in the future.

\section{CONCLUSIONS}

VC urgent care clinics to address possible COVID-19 symptoms facilitated expeditious PCP assessment while keeping potentially contagious patients outside of high-risk health care environments. Streamlining and optimizing clinical VC assessments will be imperative to future management of COVID19 and potentially to other future infectious pandemics. This includes development of templated notes incorporating counseling regarding appropriate isolation, questions about high-contact occupations, and goals of care discussions.

\section{Acknowledgment \\ The authors thank Robert F. Walsh, MHA.}

\section{Author Affiliations}

Rebecca Brienza and Juliette Spelman are Primary Care Physicians, Christopher Ruser is Chief of Primary Care, all at Veterans Affairs Connecticut Healthcare System in West Haven. Rebecca Brienza and Christopher Ruser are Associate Professors, Juliette Spelman is an Assistant Professor, and Kelly Hager is an Internal Medicine Resident, all at Yale School of Medicine in Connecticut.

\section{Author disclosures}

The authors report no actual or potential conflicts of interest with regard to this article. 


\section{Disclaimer}

The opinions expressed herein are those of the authors and do not necessarily reflect those of Federal Practitioner, Frontline Medical Communications Inc., the US Government, or any of its agencies.

\section{References}

1. Bashshur RL, Howell JD, Krupinski EA, Harms KM, Bashshur $\mathrm{N}$, Doarn CR. The empirical foundations of telemedicine interventions in primary care. Telemed J E Health. 2016;22(5):342375. doi:10.1089/tmj.2016.0045

2. Centers for Disease Control and Prevention. Using telehealth to expand access to essential health services during the COVID-19 pandemic. Updated June 10, 2020. Accessed August 20, 2021. https://www.cdc.gov/coronavirus/2019-ncov /hcp/telehealth.html

3. Harvey JB, Valenta S, Simpson K, Lyles M, McElligott J. Utilization of outpatient telehealth services in parity and nonparity states 2010-2015. Telemed J E Health. 2019;25(2):132-136. doi:10.1089/tmj.2017.0265

4. Dorsey ER, Topol EJ. State of telehealth. N Engl J Med. 2016;375(2):154-161. doi:10.1056/NEJMra1601705

5. Rockwell KL, Gilroy AS. Incorporating telemedicine as part of COVID-19 outbreak response systems. Am J Manag Care. 2020;26(4):147-148. doi:10.37765/ajmc.2020.42784

6. Centers for Disease Control and Prevention. Healthcare facility guidance. Updated April 17, 2021. Accessed August 20, 2021. https://www.cdc.gov/coronavirus/2019-ncov/hcp /clinical-care.html

7. US Department of Health and Human Services, Health Resources and Services Administration. Policy changes during COVID-19. Accessed August 20, 2021. https://telehealth.hhs .gov/providers/policy-changes-during-the-covid-19-public -health-emergency

8. Coronavirus Preparedness and Response Supplemental Appropriation Act of 2020. 134 Stat. 146. Published February 2, 2021. Accessed August 20, 2021. https://www.govinfo.gov /content/pkg/CREC-2021-02-02/html/CREC-2021-02-02 -pt1-PgS226.htm

9. US Department of Health and Human Services. Notification of enforcement discretion for telehealth remote communications during the COVID-19 nationwide public health emergency. Updated January 20, 2021. Accessed August 20 ,
2021. https://www.hhs.gov/hipaa/for-professionals/special -topics/emergency-preparedness/notification-enforcement -discretion-telehealth/index.html

10. Centers for Medicare and Medicaid Services. Coverage and payment related to COVID-19 Medicare. 2020. Published March 23, 2020. Accessed August 20, 2021. https://www .cms.gov/files/document/03052020-medicare-covid-19-fact -sheet.pdf

11. American Telemedicine Association. ATA commends 2020 Congress for giving HHS authority to waive restrictions on telehealth for Medicare beneficiaries in response to the COVID-19 outbreak [press release]. Published March 5, 2020. Accessed August 20, 2021. https://www .americantelemed.org/press-releases/ata-commends -congress-for-waiving-restrictions-on-telehealth-for -medicare-beneficiaries-in-response-to-the-covid -19-outbreak

12. Hollander JE, Carr BG. Virtually perfect? Telemedicine for Covid-19. N Engl J Med. 2020;382(18):1679-1681. doi:10.1056/NEJMp2003539

13. Khairat $S$, Meng $C, X u Y$, Edson B, Gianforcaro R. Interpreting COVID-19 and Virtual Care Trends: Cohort Study. JMIR Public Health Surveill. 2020;6(2):e18811. Published 2020 Apr 15. doi:10.2196/18811

14. Ferguson JM, Jacobs J, Yefimova M, Greene L, Heyworth L, Zulman DM. Virtual care expansion in the Veterans Health Administration during the COVID-19 pandemic: clinical services and patient characteristics associated with utilization. J Am Med Inform Assoc. 2021;28(3):453-462. doi:10.1093/jamia/ocaa284

15. Baum A, Kaboli PJ, Schwartz MD. Reduced in-person and increased telehealth outpatient visits during the COVID19 Pandemic. Ann Intern Med. 2021;174(1):129-131. doi:10.7326/M20-3026

16. Spelman JF, Brienza R, Walsh RF, et al. A model for rapid transition to virtual care, VA Connecticut primary care response to COVID-19. J Gen Intern Med. 2020;35(10):30733076. doi:10.1007/s11606-020-06041-4

17. Der-Martirosian C, Chu K, Dobalian A. Use of telehealth to improve access to care at the United States Department of Veterans Affairs during the 2017 Atlantic hurricane season [published online ahead of print, 2020 Apr 13]. Disaster Med Public Health Prep. 2020;1-5. doi:10.1017/dmp.2020.88 